International Journal of Pure and Applied Mathematics

Volume 113 No. 1 2017, 1-5

ISSN: 1311-8080 (printed version); ISSN: 1314-3395 (on-line version)

url: http://www.ijpam.eu

doi: 10.12732/ijpam.v113i1.1

ijpam.eu

\title{
A NEW PROOF OF \\ THE NAGATA-SMIRNOV METRIZATION THEOREM
}

\author{
Athanasios Andrikopoulos \\ Department of Computer Engineering \\ and Informatics \\ University of Patras \\ Panepistimioupoli Patron 265 04, GREECE
}

\begin{abstract}
In this note, I present a new, elementary proof of the Nagata-Smirnov metrization theorem.
\end{abstract}

AMS Subject Classification: 54E35

Key Words: metric, uniformity, $\sigma$-locally finite base, metrizability

The Nagata-Smirnov metrization theorem in topology characterizes the metrizability of a topological space (see [3],[5] and [6]). There exist several equivalent formulations of this theorem in the literature, but are rather complicated. In this note, based on Rudin's proof of Stone's result on paracompactness (see [4]), I offer a simple and elementary proof of this fundamental theorem. For the terminology used here, see, e.g., books [1] and [2].

Theorem. A topological space $(X, \tau)$ is metrizable if and only if it is regular and has a $\sigma$-locally finite base.

$\begin{array}{lr}\text { Received: } & \text { September 11, } 2015 \\ \text { Revised: } & \text { February 17, } 2017 \\ \text { Published: } & \text { February 28, } 2017\end{array}$

(c) 2017 Academic Publications, Ltd. url: www.acadpubl.eu 
Proof. Let $(X, \tau)$ be a metrizable space and let $d$ be a metric for $\tau$. Given $m \in \mathbb{N}$, let the open covering $\mathcal{C}_{m}=\left\{B_{d}\left(t, \frac{1}{m}\right) \mid t \in X\right\}$. By Well-Ordering Principle, there exists a well-ordered set $I$ which serves as an index set for the elements of $X$, so we may write $\mathcal{C}_{m}=\mathcal{C}_{m}^{i}$. Fix an $m \in \mathbb{N}$. For $i \in I, n \in \mathbb{N}$ define, inductively over $n$

$$
\begin{gathered}
D_{m, n, i}=\left\{x \in X \mid B\left(x, \frac{3}{2^{n}}\right) \subset \mathcal{C}_{m}^{i}, x \notin \bigcup_{j<i} \mathcal{C}_{m}^{j} \cup \bigcup_{j \in I, k<n} V_{m, k, j},\right. \\
V_{m, n, i}=\bigcup_{m \in \mathbb{N}}\left(\bigcup_{x \in D_{m, n, i}} B\left(x, \frac{1}{2^{n}}\right)\right) .
\end{gathered}
$$

Let

$$
\mathcal{V}_{\mu}=\bigcup_{m=1}^{\mu} \bigcup_{n=1}^{\mu} \bigcup_{i \in I} V_{m, n, i}
$$

We prove that $\left(\mathcal{V}_{\mu}\right)_{\mu \in \mathbb{N}}$ is a $\sigma$-locally finite base. Fix $m^{*}, n^{*} \in \mathbb{N}$. We first show that the family $\left\{V_{m^{*}, n^{*}, i} \mid i \in I\right\}$ is locally finite. For $x \in X$ let $i^{*}=\min \{i \in$ $\left.I \mid x \in \mathcal{C}_{m^{*}}^{i}\right\}$ and choose $n \in \mathbb{N}$ such that $B\left(x, \frac{3}{2^{n}}\right) \subset \mathcal{C}_{m^{*}}^{i^{*}}$. Then, by (1), either $x \in V_{m^{*}, k, j}$ for some $j \in I$ and $k<n$ or we have $x \in V_{m^{*}, n, i^{*}}$. Therefore,

$$
\left\{V_{m^{*}, n, i} \mid n \in \mathbb{N}, i \in I\right\}
$$

covers $X$. Let $x \in X$ and define $\widehat{i}=\min \left\{i \in I \mid x \in \bigcup_{n \in \mathbb{N}} V_{m^{*}, n, i}\right\}$. Then, we can choose $n, k \in \mathbb{N}$ such that $B\left(x, \frac{1}{2^{k}}\right) \subset V_{m^{*}, n, \hat{i}}$. We prove that $B\left(x, \frac{1}{2^{n+k}}\right)$ intersects $V_{m^{*}, n^{*}, i}$ for at most one $i \in I$. We have two cases to consider: $(\alpha)$ $n^{*} \geq n+k ;(\beta) n^{*}<n+k$. To prove case $(\alpha)$ suppose that $y \in D_{m^{*}, n^{*}, i}$. Since $n^{*}>n,(1)$ implies that $y \notin V_{m^{*}, n, \hat{i}}$. Therefore, $B\left(x, \frac{1}{2^{k}}\right) \subset V_{m^{*}, n, \widehat{i}}$ implies that $d(x, y) \geq \frac{1}{2^{k}}$. Now, $z \in B\left(x, \frac{1}{2^{n+k}}\right) \cap B\left(x, \frac{1}{2^{n^{*}}}\right)$ would imply $d(x, y) \leq d(x, z)+d(z, y)<\frac{1}{2^{n+k}}+\frac{1}{2^{n^{*}}} \leq \frac{1}{2^{k+1}}+\frac{1}{2^{k+1}}=\frac{1}{2^{k}}$ which is a contradiction. Thus, $B\left(x, \frac{1}{2^{n+k}}\right) \cap B\left(y, \frac{1}{2^{n^{*}}}\right)=\emptyset$. It follows $B\left(x, \frac{1}{2^{n+k}}\right) \cap$ $V_{m^{*}, n^{*}, i}=\emptyset$. To prove $(\beta)$, let $y \in V_{m^{*}, n^{*}, i}$. Since $x \in V_{m^{*}, n, \widehat{i}}$ there are $x^{\prime}, y^{\prime}$ such that $x \in B\left(x^{\prime}, \frac{1}{2^{n^{*}}}\right) \subset V_{m^{*}, n, \hat{i}}, y \in B\left(y^{\prime}, \frac{1}{2^{n}}\right) \subset V_{m^{*}, n^{*}, i}, B\left(x^{\prime}, \frac{3}{2^{n^{*}}}\right) \subset$ $\mathcal{C}_{m^{*}}^{\widehat{i}}$ and $B\left(y^{\prime}, \frac{3}{2^{n}}\right) \subset \mathcal{C}_{m^{*}}^{i}$. Suppose that $\widehat{i}<i$. Then, by (1), $y^{\prime} \notin \mathcal{C}_{m^{*}}^{\widehat{i}}$. 
This implies $d\left(x^{\prime}, y^{\prime}\right) \geq \frac{3}{2^{n^{*}}}$ and with the triangle inequality we have $\frac{3}{2^{n^{*}}} \leq$ $d\left(x^{\prime}, y^{\prime}\right) \leq d\left(x^{\prime}, x\right)+d(x, y)+d\left(y, y^{\prime}\right)<d(x, y)+\frac{1}{2^{n^{*}}}+\frac{1}{2^{n}}$. If $n^{*}>n$, we have nothing to prove because of $(\alpha)$. Suppose that $n>n^{*}$. Then, we have $d(x, y)>\frac{1}{2^{n^{*}}}>\frac{1}{2^{n+k}}$. Similarly, if $i<\widehat{i}$ we have that $d(x, y)=d(y, x)>\frac{1}{2^{n}}>$ $\frac{1}{2^{n+k}}$. Therefore, $B\left(x, \frac{1}{2^{n+k}}\right) \cap V_{m^{*}, n^{*}, j}=\emptyset$ whenever $j \neq \hat{i}$. It follows that $B\left(x, \frac{1}{2^{n+k}}\right)$ intersects at most $V_{m^{*}, n^{*}, \hat{i}}$. Therefore, for each $m, n \in \mathbb{N}$ the family $\left\{V_{m, n, i} \mid i \in I\right\}$ is locally finite. Since $\mathcal{V}_{\mu}$ is the finite union of locally finite families is locally finite. To prove that $\left(\mathcal{V}_{\mu}\right)_{\mu \in \mathbb{N}}$ is a $\sigma$-locally finite base it remains to show that $\bigcup_{\mu \in \mathbb{N}} \mathcal{V}_{\mu}$ is a base for $\tau$. We prove that given $O \in \tau$ and $x \in O$, there is an element $V_{m, n, i}$ containing $x$ and contained in $O$. Choose $m \in \mathbb{N}$ so that $\mathcal{C}_{m}^{j}=B\left(x, \frac{1}{m}\right) \subset O$. By (4) for $m^{*}=5 m$ we have that $\left\{V_{5 m, n, i} \mid m, n \in \mathbb{N}, i \in I\right\}$ is a covering of $X$. Then, $x \in V_{5 m, n, i} \subseteq \mathcal{C}_{5 m}^{i}=B\left(t, \frac{1}{5 m}\right)$ for some $n \in \mathbb{N}, i \in I$ and $t \in X$. Suppose that $\gamma \in V_{5 m, n, i}$. Then, since $V_{5 m, n, i}^{5 m}$ has diameter at most $\frac{2}{5 m}$ we have $d(x, \gamma) \leq d(x, t)+d(t, \gamma)<\frac{2}{5 m}+\frac{2}{5 m}=\frac{4}{5 m}<\frac{1}{m}$. It follows that $x \in V_{5 m, n, i} \subset B\left(x, \frac{1}{m}\right) \subset O$.

To prove the converse, suppose that $(X, \tau)$ admits a $\sigma$-locally finite base $\widetilde{\mathcal{V}}=\left\{\mathcal{V}_{n} \mid n \in \mathbb{N}\right\}$. We can assume that each $\mathcal{V}_{n}$ contains $X$ and the empty set. Fix $n \in \mathbb{N}$. For each $x \in X$, let $M_{x}^{n}=\bigcap\left\{A \mid x \in A, A \in \mathcal{V}_{n}\right\}, N_{x}^{n}=$ $\bigcap\left\{X \backslash c l_{\tau} A \mid x \in X \backslash c l_{\tau} A\right.$ and $\left.A \in \mathcal{V}_{n}\right\}$ and $U_{n}=\left\{M_{x}^{n} \cap N_{x}^{n} \times M_{x}^{n} \cap N_{x}^{n} \mid x \in X\right\}$ $\left(c l_{\tau} A\right.$ denotes the closure of $A$ with respect to $\left.\tau\right)$. The family $\left(U_{n}\right)_{n \in \mathbb{N}}$ is nested since for all $m, n \in \mathbb{N}, \mathcal{V}_{m} \subseteq \mathcal{V}_{n}$ implies $U_{m} \supseteq U_{n}$. Since $\widetilde{\mathcal{V}}$ is a $\sigma$-locally finite base, $M_{x}^{n}, N_{x}^{n}$ are open and for each $x \in X, U_{n}(x)$ is a neighborhood system at $x$ which generates $\tau$. Let $x \in X$ and $n \in \mathbb{N}$. Since $(X, \tau)$ is regular and $\left(U_{n}\right)_{n \in \mathbb{N}}$ is nested, we may choose $m \in \mathbb{N}(m>n)$ and $B \in \mathcal{V}_{m}$ so that $x \in U_{m}(x) \subseteq B \subseteq c l_{\tau} B \subseteq U_{n}(x)$. Then, we have $U_{m}^{2}(x)=U_{m}\left(U_{m}(x)\right)=$ $\bigcup\left\{M_{y}^{m} \cap N_{y}^{m} \mid M_{y}^{m} \cap N_{y}^{m} \cap U_{m}(x) \neq \emptyset\right\}$. Let $y \in U_{n}(x)$. Then, $M_{y}^{m} \subseteq U_{n}(x)$. $y \in X$

If $y \notin U_{n}(x)$, then $y \in X \backslash c l_{\tau} B$ and $N_{y}^{m} \cap B=\emptyset$. It follows that $N_{y}^{m} \cap U_{m}(x)=\emptyset$ which implies that $U_{m}^{2}(x) \subseteq U_{n}(x)$. Inductively we define a sequence $\left(\mathcal{W}_{n}\right)_{n \in \mathbb{N}}$ of neighborhnets of $X$ as follows: $\mathcal{W}_{1}=U_{1}^{2}$ and for each $n \in \mathbb{N}$

$$
\mathcal{W}_{n+1}=\bigcup\left\{U_{m}(x) \times U_{m}(x) \mid m \geq n+1, x \in X \text { and } U_{m}^{3}(x) \times U_{m}^{3}(x) \subseteq \mathcal{W}_{n}\right\}
$$


It is easy to check that $\mathcal{W}_{n+1}^{2} \subseteq \mathcal{W}_{n}$. It follows that $\left(\mathcal{W}_{n}\right)_{n \in \mathbb{N}}$ is a base for a uniformity which is compatible with $\tau\left(\mathcal{W}_{n} \subseteq U_{n}^{2}\right)$. We define the functions $f$ and $d$ on $X \times X$ by setting: $f(x, y)=\inf \left\{\frac{1}{n} \mid n \in \mathbb{N}\right.$ and $\left.y \in \mathcal{W}_{n}^{2}(x)\right\}$ and $d(x, y)=\inf \left\{\sum_{i=0}^{n} f\left(x_{i}, x_{i+1}\right) \mid n \in \mathbb{N}, x_{i} \in X\right.$ for each $i, x_{0}=x$ and $\left.x_{n+1}=y\right\}$. Clearly, by definition, $d$ is a metric on $X$. It remains to prove that $\tau_{d}=\tau$. We first prove that $d(x, y) \leq f(x, y) \leq 2^{n} d(x, y)$ for each $x, y \in X$. The first inequality is evident. To see the second one, we first show that

$$
f(x, y) \leq 2(f(x, z)+f(z, y)) \text { for all } x, z, y \in X
$$

The inequality clearly holds in case $\max (f(x, z), f(z, y))=1$. Suppose that $\max (f(x, z), f(z, y)) \leq \frac{1}{2}$. Then, there exists $n \in \mathbb{N}$ such that $\frac{1}{2^{n+1}}<\max (f(x, z)$,

$f(z, y))=\frac{1}{2^{n}}$. Without loss of generality, we assume that $d(x, z)=\frac{1}{2^{\mu}} \leq$ $\frac{1}{2^{n}}$ and $d(z, y)=\frac{1}{2^{n}}$. It follows that $z \in \mathcal{W}_{\mu}^{2}(x) \subseteq \mathcal{W}_{n}^{2}(x)(n<\mu)$ and $y \in \mathcal{W}_{n}^{2}(z)$. Hence, $y \in \mathcal{W}_{n}^{4}(x) \subseteq \mathcal{W}_{n-1}^{2}(x)$. Therefore, $f(x, y) \leq \frac{1}{2^{n-1}}=$ $\frac{2}{2^{n}}=2 \max (f(x, z), f(z, y)) \leq 2(f(x, z)+f(z, y))$. We now prove that for all $x_{0}, x_{1}, \ldots, x_{n+1} \in X$ we have

$$
f\left(x_{0}, x_{n+1}\right) \leq 2^{n}\left\{\sum_{i=0}^{n} f\left(x_{i}, x_{i+1}\right) \mid n \in \mathbb{N}, x_{i} \in X\right\} .
$$

For $n=1$, the inequality reduces to $f\left(x_{0}, x_{2}\right) \leq 2 f\left(x_{0}, x_{1}\right)+2 f\left(x_{1}, x_{2}\right)$ and this holds by (5). Assume that the result has been proved for $n=k$. Then for all $x_{0}, x_{1}, \ldots, x_{k+1} \in X$ we have

$$
f\left(x_{0}, x_{k}\right) \leq 2^{k}\left\{\sum_{i=0}^{k} f\left(x_{i}, x_{i+1}\right) \mid k \in \mathbb{N}, x_{i} \in X\right\}
$$

But then, by using (5) we have $f\left(x_{0}, x_{k+1}\right) \leq 2 f\left(x_{0}, x_{k}\right)+2 f\left(x_{k}, x_{k+1}\right) \leq$ $2^{k+1}\left\{\sum_{i=0}^{n} f\left(x_{i}, x_{k+1}\right) \mid k \in \mathbb{N}, x_{i} \in X\right\}+2 f\left(x_{k}, x_{k+1}\right) \leq 2^{k+1}\left\{\sum_{i=0}^{k+1} f\left(x_{i}, x_{k+1}\right) \mid k \in\right.$ $\left.\mathbb{N}, x_{i} \in X\right\}$. Therefore, (6) holds. So we have that $f(x, y) \leq 2^{n} d(x, y)$ as required. To prove that $\tau \subseteq \tau_{d}$, let $y \in B_{d}\left(x, \frac{1}{2^{2 n}}\right)$. Then we have that $f(x, y) \leq$ 
$2^{n} d(x, y) \leq 2^{n} \frac{1}{2^{2 n}}$, in other words, that $y \in \mathcal{W}_{n}^{2}(x) \subseteq \mathcal{W}_{n-1}(x)$. We have shown that $B_{d}\left(x, \frac{1}{2^{2 n}}\right) \subset \mathcal{W}_{n-1}(x)$. To prove that $\tau_{d} \subseteq \tau$, let $y \in \mathcal{W}_{n}^{2}(x)$. Then we have $d(x, y) \leq f(x, y) \leq \frac{1}{2^{n}}<\frac{1}{2^{n-1}}$ It follows that $\mathcal{W}_{n}(x) \subseteq \mathcal{W}_{n}^{2}(x) \subset B_{d}\left(x, \frac{1}{2^{n-1}}\right)$ which completes the proof.

\section{References}

[1] J. Kelley, General Topology, D. Van Nostrand Company Inc., Toronto-New York-London, 1955.

[2] J. Munkres, Topology: A First Course, Prentice-Hall Inc., Englewood Cliffs, N.J., 1975.

[3] J. Nagata, On a necessary and sufficient condition for metrizability, J. Inst., Polytech. Osaka City Univ., Ser. A Math. 1 (1950), 93-100.

[4] M.E. Rudin, A new proof that metric spaces are paracompact, Proc. Amer. Math. Soc., 20 (1969), 603.

[5] J.M. Smirnov, A necessary and sufficient condition for metrizability of a topological space, Doklary Akad. Nauk SSSR (N.S), 77 (1951), 197-200.

[6] J. Williams, Locally uniform spaces, Trans. Amer. Math. Soc., 168 (1972), 435-469. 
